\title{
The impact of exercises on the physical and psychological health of hearing impaired
}

\author{
Nikolay Ryzhkin ${ }^{1}$, Anna Ivanova $^{1 *}$, Olga Brovashova $^{1}$ and Tatiana Tumasyan ${ }^{1}$ \\ ${ }^{1}$ Don State Technical University, 1, Gagarin Sq., 344003, Rostov-on-Don, Russia
}

\begin{abstract}
This article discusses the features of the construction of the educational process in physical culture for children of primary school age with hearing impairments. This problem is relevant due to the fact that at present the health of children and their physical development are of paramount importance in the development of various social programs, including physical education.
\end{abstract}

\section{Introduction}

Currently, the number of children with various pathologies has increased throughout the world. Deterioration of the environment, genetic changes, exposure to toxic and chemical substances lead to the pathology of newborns. Today, one of the priority directions of the internal policy of our state is both concern for the health of the nation in general, and for the health of the younger generation in particular.

In the presented work, theoretical and methodological material on the physical qualities of children and the methods of their upbringing is collected and generalized, the physiology of deaf and hard of hearing children is considered in detail. In addition, the categories of children were identified depending on the degree of hearing impairment and, accordingly, depending on the characteristics of their perception of sound, visual, sensory and signal information from the world around them. The most acceptable types of physical culture and sports activities for children with hearing impairments are highlighted, the peculiarities of physical culture lessons for the development of physical qualities are shown [6, p. 54]. Practical, visual and verbal methods of the process of teaching motor actions of deaf and hard of hearing children are considered, taking into account their physiological characteristics.

Purpose of the research: to scientifically substantiate the presented curriculum on physical culture for deaf and hard of hearing students of the 1st grade, taking into account the individual typological characteristics.

The hypothesis of the study was the assumption that the revealed distinctive features of the process of physical education of deaf and hearing impaired children, taking into account their physiological characteristics and the peculiarities of the manifestation of motor abilities in hearing impaired children of $7-8$ years old, will make it possible to develop and experimntally substantiate a physical education curriculum for deaf and hard of hearing students of the 1st grade. Alive organisms are in constant complex dynamic interaction with the environment. In the scientific definition of the concepts of "organism" and "environment"

\footnotetext{
* Corresponding author: lagosha58@mail.ru
} 
should be considered as a single whole, as a single functional system. The basis for the normal existence and preservation of a single functional system is the constancy of the internal environment. When various factors act on the body, homeostasis changes, as a result adaptive processes arise in the body. They are formed and aimed at restoring and compensating for impaired functions in order to maintain normal life.

There are different types of adaptation to various environmental factors, including physical activity. The peculiarities of the adaptation of the human body to the acting factors depend on the individual characteristics of the person, the level of his physical development and physical performance, which is one of the components of the state of health.

Adolescence is the most difficult stage of ontogenesis when the child's body reaches biological maturity. Therefore, intensive morphological and functional transformations of organs and systems during puberty, on the one hand, cause a greater sensitivity of adolescents to any changes in environmental factors, and on the other hand, they turn the processes of growth and differentiation and, therefore, the final results of development into one of the main targets or indicators of the influence of the environment.

In the general problem of the growth and development of the younger generation, it is of particular interest to study the tendencies of this process in recent years, which, to a certain extent, is associated with the acceleration observed over decades.

The study of the functional capabilities of a person at different stages of his individual development is important for the further development of the fundamental foundations of the theory of ontogenesis and age periodization, as well as for the practice of medical control and physical education of the younger generation.

The study of the dynamics of the development of anthropometric indicators makes it possible to state the relative unevenness of the development of the length and weight of the body of schoolchildren both in different periods of life, and when comparing these indicators with each other. It has been established that the genetic need for different levels of movement depends on the typological characteristics of the child's nervous system. At the same time, the optimal physical development and functional state of the child's body is achieved with physical activity at 4 years old - 12-13 thousand locomotions, at 5 years old - 14-15 thousand, at 6 and $7-15-16$ and 17-18 thousand movements, respectively.

The main areas of research on this issue are:

1) development of methods for measuring physical activity (quantitative indicators of character, volume, intensity);

2) assessment of physical fitness and its relationship with health and cultural development;

3) study of the effect of various types of physical activity;

4) analysis of the level of physical activity of different age groups;

5) study of the features of the formation of physical activity and its impact on health;

6) development of new methods of conducting physical exercises with children;

7) study of the diagnostic and prognostic role of testing exercises for children.

\section{Materials and methods}

The study of the impact of physical exercises on the physical, psychological aspects of life of primary schoolchildren with varying degrees of hearing impairmnts was carried out with a group of 7-8 year old schoolchildren on the basis of schools No. 50, 110 in cooperation with the Don State Technical University (2017-2018). In total, 38 people with hearing impairments took part in the study, including 25 boys and 13 girls. This group included hearing impaired children with various impairments. The work was carried out in contact with the school doctor, taking into account the state of health of the students, data on the medical group, the structure of the defect of each child. The work was planned according to the "Program on physical culture of special schools for hearing impaired children." 
Based on the analysis and generalization of scientific and methodological literature data, the task was to identify the distinctive features of the process of physical education of deaf and hard of hearing children, taking into account their physiological characteristics, to determine the initial level of development of conditioning and coordination abilities in hearing impaired children of 7-8 years old. And also to develop and experimentally test a curriculum on physical culture for deaf and hard of hearing students of the 1st grade, taking into account the individual-typological characteristics.

Research object: the process of physical education of children with hearing impairments.

Subject of the research: the peculiarities of the construction of the educational process in physical culture for children of primary school age with hearing impairments.

Research methods: theoretical analysis and generalization of scientific and methodical literature data; dynamic pedagogical and medical supervision; pedagogical experiment; pedagogical testing; physical testing; methods of mathematical statistics.

The analysis of literary sources and documentary materials was carried out by studying the author's abstracts, articles from collections, scientific papers, educational and methodological literature, programs for preschoolers.

When analyzing literary sources, the main attention was paid to identifying such issues as the development of motor abilities in school-age children, as well as the physical education of children with disabilities.

The method of pedagogical observation was used to reveal the influence of the developed curriculum on the general functional state of children with disabilities, on the level of mastery of the technique of motor actions.

The testing method was used to determine the effectiveness of the application of the experimental program during the year for the preparedness of children.

We used the following tests:

1. Shuttle run $3 \times 10 \mathrm{~m}$ - to determine the level of coordination abilities;

2. Raising the body from a prone position, arms up, in 30 seconds. - to determine strength abilities;

3. Running 30 meters - determining the level of speed abilities;

4. Long jump from a place - determination of the level of speed-power abilities.

In the process of the pedagogical experiment, two lessons were conducted per week and one additional game lesson in the hall. In addition, the group held classes on outdoor games in the fresh air, based on our recommendations.

The research was carried out in several stages.

1. Stage: determination of control standards for children 7-8 years old. Conducting at the beginning of the school year, the fourth week of September, control standards to determine the level of development of conditioning and coordination abilities in children. Drawing up an experimental curriculum on physical culture for a group of hearing impaired children 7-8 years old.

2. Stage: testing a specialized program in the classroom with a group of hearing impaired children.

3. Stage: retesting to determine the effectiveness of this program, analysis of the results of work with the experimental group.

In the process of the pedagogical experiment, physical culture lessons, exercise therapy classes, rhythmics and extra-curricular physical culture and sports and physical culture and health-improving activities were carried out, in which correctional and educational work was carried out in all types of activities. 
Table 1. Survey of the level of development of physical qualities of children with disabilities

\begin{tabular}{|c|c|c|c|c|c|c|c|c|c|c|c|}
\hline \multirow{3}{*}{$\begin{array}{c}\text { Physic } \\
\text { al } \\
\text { abilitie } \\
\text { s }\end{array}$} & \multirow{3}{*}{$\begin{array}{c}\text { Control } \\
\text { exercises }\end{array}$} & \multicolumn{8}{|c|}{ Children with disabilities } & \multicolumn{2}{|c|}{ Software standards } \\
\hline & & \multicolumn{4}{|c|}{ Boys } & \multicolumn{4}{|c|}{ Girls } & \multirow[b]{2}{*}{ స్. } & \\
\hline & & : & $\underbrace{\vec{\Xi}}_{\tilde{\sigma}}$ & 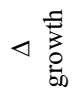 & 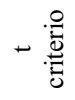 & : & $\underbrace{\vec{\Xi}}_{\vec{E}}$ & 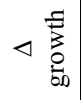 & 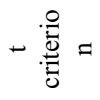 & & \\
\hline \multirow{2}{*}{ 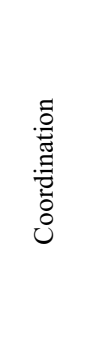 } & 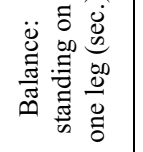 & 5,5 & 8,4 & 2,88 & 14,4 & 7,3 & 11,5 & 2,79 & 9,33 & $\begin{array}{l}8- \\
10\end{array}$ & $9-12$ \\
\hline & 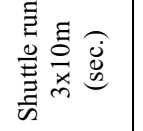 & $\begin{array}{c}15, \\
3\end{array}$ & $\begin{array}{c}13, \\
9\end{array}$ & 1,47 & 8,21 & 15,9 & 13,2 & 4,12 & 7,88 & 11,5 & 12,1 \\
\hline 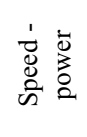 & 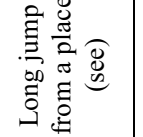 & 118 & 135 & 18 & $\begin{array}{c}10,9 \\
6\end{array}$ & 123 & 136 & $\begin{array}{c}13,1 \\
2\end{array}$ & 8,79 & 129 & 119 \\
\hline 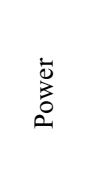 & 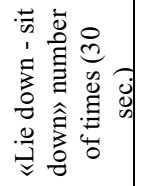 & $\begin{array}{c}11, \\
3\end{array}$ & $\begin{array}{c}16, \\
2\end{array}$ & 4,88 & $\begin{array}{c}12,8 \\
6\end{array}$ & 11,2 & 16,5 & 5,25 & 14,34 & 17,5 & 15,5 \\
\hline \multirow{2}{*}{ 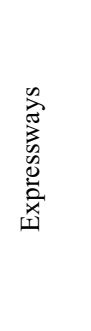 } & 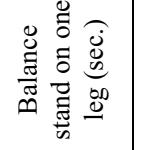 & 5,5 & 8,4 & 2,88 & 14,4 & 7,3 & 11,5 & 2,79 & 9,33 & $\begin{array}{l}8- \\
10\end{array}$ & $9-12$ \\
\hline & 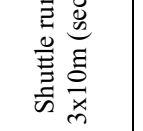 & $\begin{array}{c}15, \\
3\end{array}$ & $\begin{array}{c}13, \\
9\end{array}$ & 1,47 & 8,21 & 15,9 & 13,2 & 4,12 & 7,88 & 11,5 & 12,1 \\
\hline
\end{tabular}

\section{Results}

The system of selected dynamic exercises influences the hearing aids of 7-8 years old children in many ways. During the study, it was noticed that the average level of development of all physical abilities of children with disabilities using the experimental program increased significantly. 


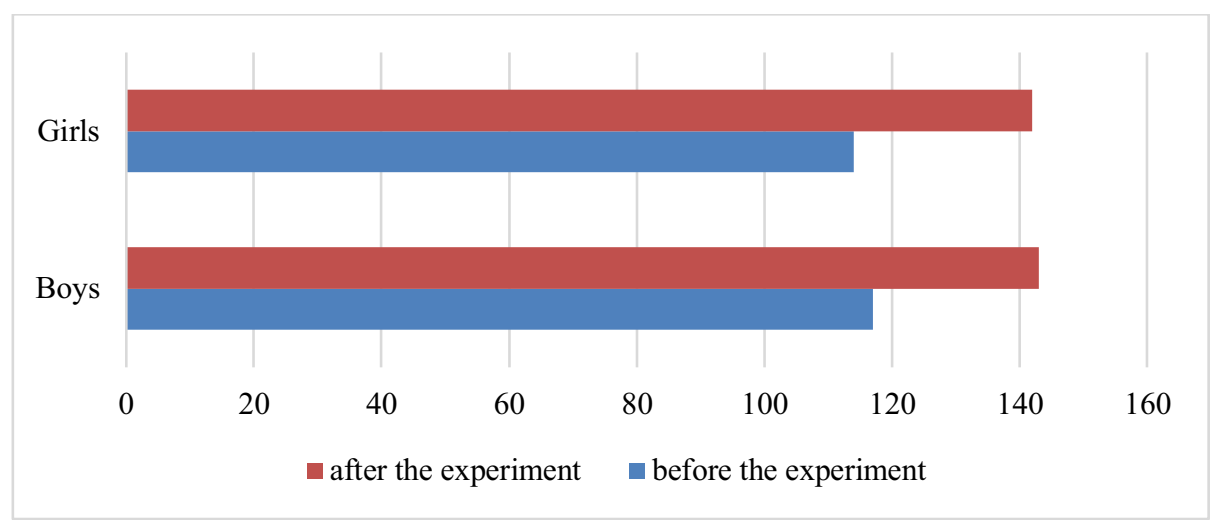

Fig. 1. Dynamics of test results in the process of conducting a pedagogical experiment. Standing long jump (mm)

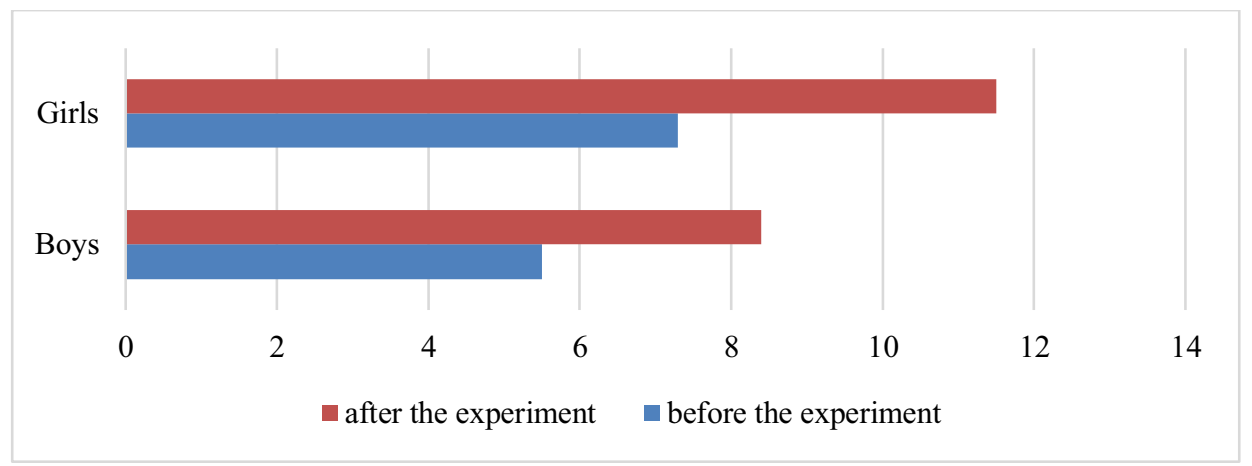

Fig. 2. Dynamics of test results in the process of conducting a pedagogical experiment. Static balance holding time - standing on one leg (sec.)

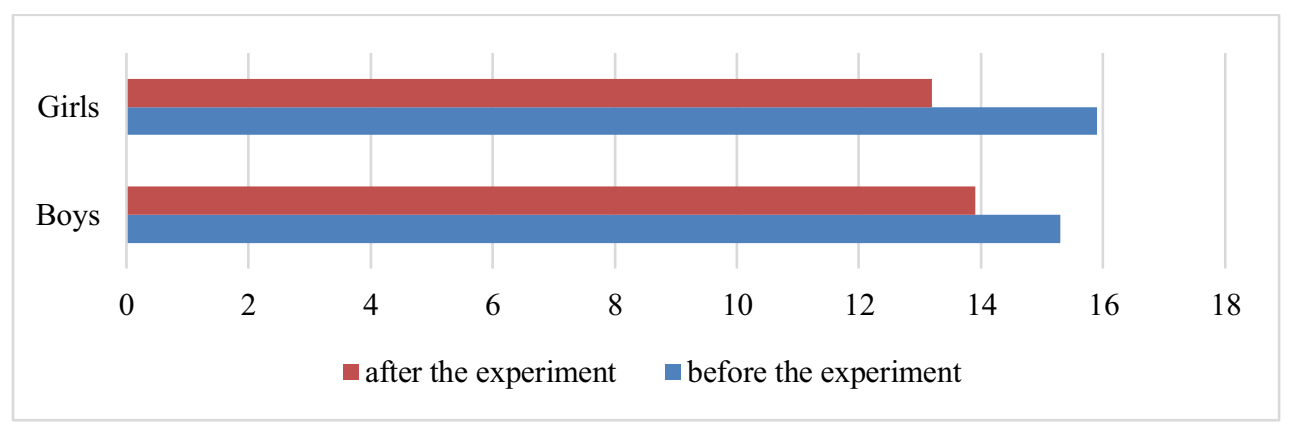

Fig. 3. Dynamics of test results in the process of conducting a pedagogical experiment. Shuttle run $3 \times 10 \mathrm{~m}(\mathrm{sec}$. 


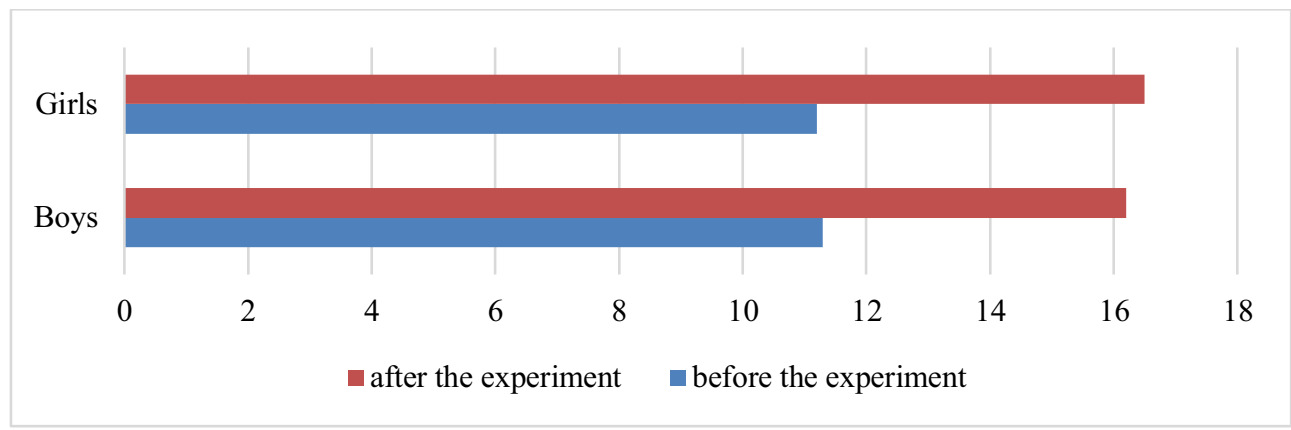

Fig. 4. Dynamics of test results in the process of conducting a pedagogical experiment. "Lie down - sit down" number of times $(30 \mathrm{sec}$.)

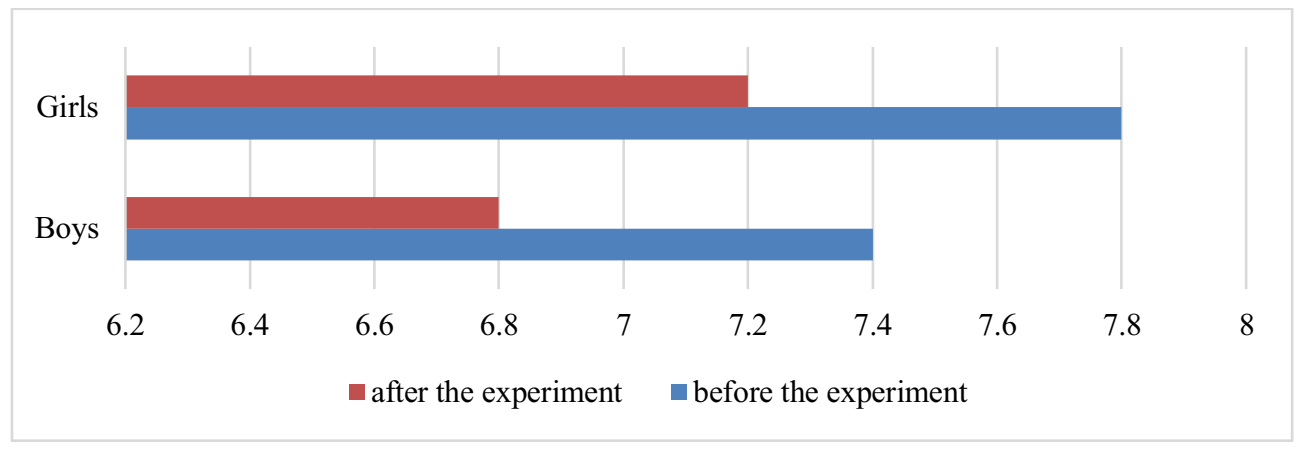

Fig. 5. Dynamics of test results in the process of conducting a pedagogical experiment. Running $30 \mathrm{~m}$ (sec.)

The greatest gains are noted in the long jump from the spot. Long jump in girls in primary testing was higher than the average program standards, and after training in the experimental program it increased by an average of $13.1 \mathrm{~cm}$.In boys, in the primary testing, the indicator was lower than the average control standard, with repeated testing, the result increased on average by $18 \mathrm{~cm}$. Thus, we can state the presence of reliable statistical differences between the initial and final results.

In the manifestation of coordination abilities - in the test «balance standing on one leg», the girls achieved significant improvement, showing the result of $11.5 \mathrm{~s}-$ the result of the upper limit of the average indicator. In boys, the same test reached the average level of program requirements. The calculation of arithmetic mean values, standard deviations and comparison of the boundaries of the confidence intervals show statistically significant differences between the indices of the initial and final tests in the groups of boys and girls. In another test «shuttle run $3 \times 10 \mathrm{~m}$ » similar changes were recorded during the period of the pedagogical experiment.

Indicators of speed abilities in children with disabilities lag behind the program standards, although they have a statistically significant increase in results.

The results of the research lead to a logical statement: this program has a positive effect on the growing body of the child.

The level of development of physical qualities of children with disabilities began to meet the accepted program requirements.

The results of preliminary tests showed that the average level of development of the motor abilities of hearing-impaired children lags behind the program standards, with the exception of the long jump in girls. The results of pedagogical observations made it possible to reveal some features in the regulation of movements in students of a special school. The data 
obtained indicate that these children have impaired spatial orientation of movements and there are deviations in the regulation of the strength of movements, which is determined by the degree of development of the motor analyzer, although muscle strength plays an important role in jumping.

Repeated testing showed that the average level of development of motor abilities in children with disabilities using a special program has increased significantly.

The results of the study indicate that the experimental program has a positive effect on the indicators of the development of the motor abilities of 1st grade pupils with hearing impairments, since the level of development of the physical qualities of children with disabilities began to correspond to the accepted program requirements.

When determining the level of development of the conditioning and coordination abilities of children of 7-8 years old with disabilities, one can see a noticeable lag of children in all indicators from the average control standards. The exception is the performance of girls in the long jump from the spot, which slightly exceeds the average results of the program.

For the development of motor abilities in hearing-impaired children, a working curriculum on physical culture for 1st grade students with disabilities was developed on the basis of the «Program on physical culture of special schools for hearing-impaired children», taking into account the characteristics of the state of those involved.

The impact on children of 7-8 years of age with disabilities during the school year of the experimental program with repeated testing shows that this program provides a significant increase in results in all tests reflecting the coordination and conditioning abilities of schoolchildren.

The most effective content of physical education of hearing-impaired students is a combination of basic gymnastics, games, exercise therapy exercises and rhythmic elements. The means of physical education in such a combination effectively contribute to the general physical development of students and the correction of defects in their physical condition. The use of these exercises and influences should be carried out at the correct dosage, taking into account individually age, pathological and psychological, and physical characteristics, not only in physical education lessons, but also outside school hours.

\section{Discussions}

The main condition for the selection of all tasks is that they must have a cognitive and developmental function, as well as arouse interest in the subjects. It was necessary to pay attention to fluctuations in the subjects' interest, their fatigue using statistical and mobile tasks. If a subject with a hearing impairment refused to perform any joint task, one should not insist on its completion, but invite him to follow the correctness of the task. This facilitated his adaptation and communication with peers. Eventually, the moment came when he entered into the general group activity on his own. During the lessons, some assessments of the student's successful actions were given, but this was done unobtrusively; so that these assessments do not affect the personal characteristics of the hearing impaired, but relate to the characteristics of their behavior and communication.

\section{Conclusions}

We have conducted a study of the auditory analyzer in hearing impaired students in the range of perceived frequencies. According to the indicators in some of the subjects there was not a complete loss of hearing, there were remnants of the vital activity of the auditory analyzer. Based on the auditory examination, we built an audiogram among the indicators of perceived frequencies at the beginning and at the end of the experiment. The audiogram showed that 
after systematic training according to our method there was a slight improvement in hearing (2 dB.).

The results of the study confirm the hypothesis that people with hearing impairments, after appropriate training, can actively use many forms of physical culture and sports activities that are typical for those who do not have these disorders. Physical exercises aimed at improving the motor analyzer of hearing impaired children lead to an increase in the role and compensation of the lost functions due to hearing impairment by this analyzer.

Social integration of hearing impaired students into the environment of healthy peers is a desirable, and possibly a prerequisite for its further growth and adequate social adaptation. A complex of pedagogical methods of stimulating interest and emotional tone in the structure of joint physical culture training for hearing impaired people with hearing people has been developed, including: sound signals, light signal. As the experiment has shown, to implement the above fundamental possibility of influencing the level of social adaptation and integration of the hearing impaired, it is advisable and necessary to use physical culture and sports activities in such forms and types that correspond to the interests and needs of hearing impaired children.

The authors of the article are grateful to the rector of the DSTU B.Ch. Meskhi, for the opportunity to conduct a study at the base of the DSTU track and field arena, to the director of the Institute of Physical Culture and Sports, Mavropulo O.S. for valuable advice in planning research and recommendations for the design of the article.

\section{References}

1. E.V. Khromin, All-Russian physical education and sport GTO complex: municipal management innovations, Theory and Practice of Physical Culture 1, 3-5 (2019)

2. A.V. Voronkov, Academic physical fitness rating by GTO complex tests Theory and Practice of Physical Culture 5, 11 (2019)

3. T. J. Kauh, Building a culture of health through research: The role of the physical activity research center, Preventive Medicine 130 Article 105894 (2020)

4. S. G. Farris, Development and initial validation of the Exercise Sensitivity Questionnaire, Mental Health and Physical Activity 19, Article 100346 (2020)

5. P. S. Tandon, Short term impact of physical activity vs. sedentary behavior on preschoolers' cognitive functions, Mental Health and Physical Activity 15, 17-21 (2018)

6. A. Ivanova, Mathematical analysis of individual physical fitness test rates and functionality forecasts, Physical education: education, training 2(79), 53-56 (2019)

7. A. Ivanova, Adaptation and development of the physical culture and sports complex of the GTO in accordance with changes in society, In the collection: Innovative transformations in the field of physical culture, sports and tourism. Collection of materials of the XXI All-Russian scientific and practical conference, 287-292 (2018)

8. P. S. Tandon, Short term impact of physical activity vs. sedentary behavior on preschoolers' cognitive functions, Mental Health and Physical Activity 15, 17-21 (2018)

9. S. Rahimi, Long-term exercise from adolescence to adulthood reduces anxiety- and depression-like behaviors following maternal immune activation in offspring, Physiology \& Behavior 226, Article 113130 (2020)

10. T. Stepanova, Physical education and sports sector progress in rostov region. Theory and practice of physical culture 8, 98-99 (2018)

11. J. E. Simon, Health-related quality of life is decreased in middle-aged adults with chronic ankle instability, Journal of Science and Medicine in Sport 21(12), 1206-1209 (2018) 
12. T. Wisbey-Roth, Prescribing exercise like the medicine it is. Journal of Science and Medicine in Sport 22(1), 1 (2019)

13. B. S. Frank, Movement profile influences systemic stress and biomechanical resilience to high training load exposure, Journal of Science and Medicine in Sport 22(1), 29-34 (2019)

14. J. L. Pryor, Intermittent exercise-heat exposures and intense physical activity sustain heat acclimation adaptations, Journal of Science and Medicine in Sport 22(1), 117-122 (2019)

15. M. Shestakov, Content and ways of improving the physical education of students mastering management specialties, Scientific notes of the University. P.F. Lesgaft 5(147), 199-203 (2017)

16. N. Ryzhkin, GTO complex as the basis of physical education in DSTU, Innovative Technologies in Science and Education (ITSE-2020), E3S Web of Conferences 210, 110 (2020) DOI: 10.1051/e3sconf/202021017009 